\title{
EFFECT OF STEFAN NUMBER ON THERMOELASTIC INSTABILITIES IN UNIDIRECTIONAL SOLIDIFICATION
}

\author{
FaruK Yigit and J. R. Barber \\ Department of Mechanical Engineering and Applied Mechanics, University of Michigan, \\ Ann Arbor, MI48109-2125, U.S.A.
}

(Received 1 June 1993; and in revised form 11 January 1994)

\begin{abstract}
During the casting process, thermoelastic distortion of the partially solidified material affects the contact pressure at the solid/mold interface, which in turn can affect the thermal contact resistance, thus coupling the heat transfer and thermomechanical problems. This coupled system has the potential for instability. In this paper, the effect of Stefan number on the stability of unidirectional solidification is investigated, under the simplifying assumption that the solidified material is linear elastic. The Stefan number is a measure of the influence of thermal capacity on the solution and previous analyses have generally been restricted to the case of zero Stefan number, corresponding to a solidifying material of zero thermal capacity.

This generalization necessitates a numerical solution, which is here implemented using the finite difference method. However, since the growth of the perturbation is linear, the two-dimensional stability problem is reduced to two one-dimensional numerical problems which can be solved sequentially.

The results show that, in all cases, an initial sinusoidal perturbation grows to a maximum amplitude in the solidification front and then decays, the maximum being reached when the mean solidified layer thickness is about half the wavelength of the perturbation.

In general, increasing Stefan number has a stabilizing effect on the process. This effect is most noticeable in cases where the zero Stefan number approximation predicts substantial growth of an initial perturbation, e.g. where the thermal contact resistance is very sensitive to pressure.
\end{abstract}

\section{INTRODUCTION}

During solidification processes, long wavelength perturbations in the development of the solid/melt boundary have been observed. For example, Fig. $1^{*}$ shows a section cut from a nominally axisymmetric continuous casting billet of aluminum alloy that has been drained during the process to exhibit the development of the solidification front. The thickness of the partially solidified cylindrical shell is not uniform, but contains wavy perturbations in both axial and circumferential directions. The wavelength of these perturbations is of the order of $100 \mathrm{~mm}$, which is orders of magnitude larger than those associated with the phenomenon known as morphological or dendritic instability, and which results from changes in concentration of alloying elements during solidification.

Similar long wavelength perturbations have been reported in the literature [1-6] and are generally undesirable in the manufacturing process, since the resulting ingot may have nonuniform properties and the associated nonuniform thermal stress field may cause cracking near the surface.

Richmond et al. [7] proposed that this phenomenon might be associated with an unstable interaction between the thermal distortion of the casting and the heat transfer at the casting/mold interface. To investigate this hypothesis, they considered the solidification of a pure metal against a plane mold at which the nominally uniform heat flux is given a small spatially sinusoidal perturbation. They found that the contact pressure increased in regions of high heat flux. This would be expected to reduce the local thermal contact resistance and is an indication of a potentially unstable positive feedback system. Further confirmation was provided by $\mathrm{Li}$ and Barber [8] who analyzed the coupled problem in which the heat flux at the casting/mold interface is determined by heat flow across

* This figure is reproduced by kind permission of the Aluminum Company of America. 
a pressure-dependent thermal contact resistance. They found that unstable growth of an initial sinusoidal perturbation could occur if the resistance was sufficiently sensitive to pressure.

Both of these treatments make simplifying assumptions in order to permit a predominantly analytical solution. In particular, they assume that the solidified material is linear elastic, that it is a pure material with a unique melting point, that the material properties are independent of temperature and that the Stefan number

$$
\varepsilon=\frac{c \Delta T}{L} \ll 1
$$

where $c, L$ are the specific heat and latent heat of solidification, respectively, and $\Delta T$ is the temperature difference across the solidified layer.

These approximations clearly place severe restrictions on the accuracy of the resulting predictions, but they can be justified on the grounds that the resulting analysis retains some generality and hence permits deductions to be made about the effect of changes of material properties and operating conditions on the stability of the system. By contrast, a full numerical simulation of the system using the best available material data, apart from being very computer-intensive, would only permit results to be obtained for particular cases.

The small Stefan number approximation (1) is reasonable for the solidification of ice, but not for the solidification of metals in contact with a mold near room temperature, for which Stefan numbers are typically in the range $1<\varepsilon<3$ [9]. In Richmond's problem [7], where the heat flux at the mold is specified, $\Delta T$ and hence $\varepsilon$ increase with time from zero at the onset of solidification. Thus, the idealized solution constitutes an asymptotic limit to the correct solution at small times, but would be expected to diverge from it as time progresses.

Yigit and Barber [10] assessed the effect of the small Stefan number approximation in Richmond's problem by developing a finite difference formulation for constant but non-zero specific heat. Their results showed that increased specific heat reduces the sensitivity of the contact pressure to perturbations in heat flux and hence might be expected to exert a stabilizing effect on the coupled problem. This hypothesis is examined in the present paper, which extends the analysis of $\mathrm{Li}$ and Barber [8] to materials of finite specific heat.

The other assumptions of $\mathrm{Li}$ and Barber [8] will be retained, including that of elastic behavior of the solidified material. In this connection, we note that newly solidified material will be deposited in a state of hydrostatic stress and the stress state at any given point only deviates from this condition as solidification proceeds. Thus, even though most materials exhibit reduced yield stress and enhanced creep behavior at temperatures near the melting point, we would not anticipate inelastic behavior in the immediate vicinity of the solidification front.

\section{STATEMENT OF THE PROBLEM}

We consider the two-dimensional plane strain problem of a liquid initially at its melting temperature $T_{\mathrm{m}}$, occupying the region $y>0$ and restrained by a plane mold at $y=0$. The liquid phase is assumed to be at constant hydrostatic pressure $p$. The heat flux $Q(x, t)$ from the casting to the mold is opposed by a thermal contact resistance $R$, defined by the relation

$$
Q(x, t)=K \frac{\partial T}{\partial y}(x, 0, t)=\frac{T(x, 0, t)-T_{\text {mold }}}{R},
$$

where $K$ is the thermal conductivity, $T(x, y, t)$ is temperature and $T_{\text {mold }}$ is the temperature of the rigid mold, which can be taken to be zero without loss of generality.

Physical causes of the resistance $R$ include the presence of air gaps and inclusions of materials of poor conductivity at the interface. Experimental and theoretical investigations of the conduction of heat between contacting solids show that the contact resistance is very sensitive to the local contact pressure $P(x, t)$, probably because increased contact pressure increases the proportion of the interface over which the solids are in intimate contact. The resistance $R$ is therefore assumed to be a continuous and differentiable function of $P$, but no assumptions arc made about the precise nature of this function. 


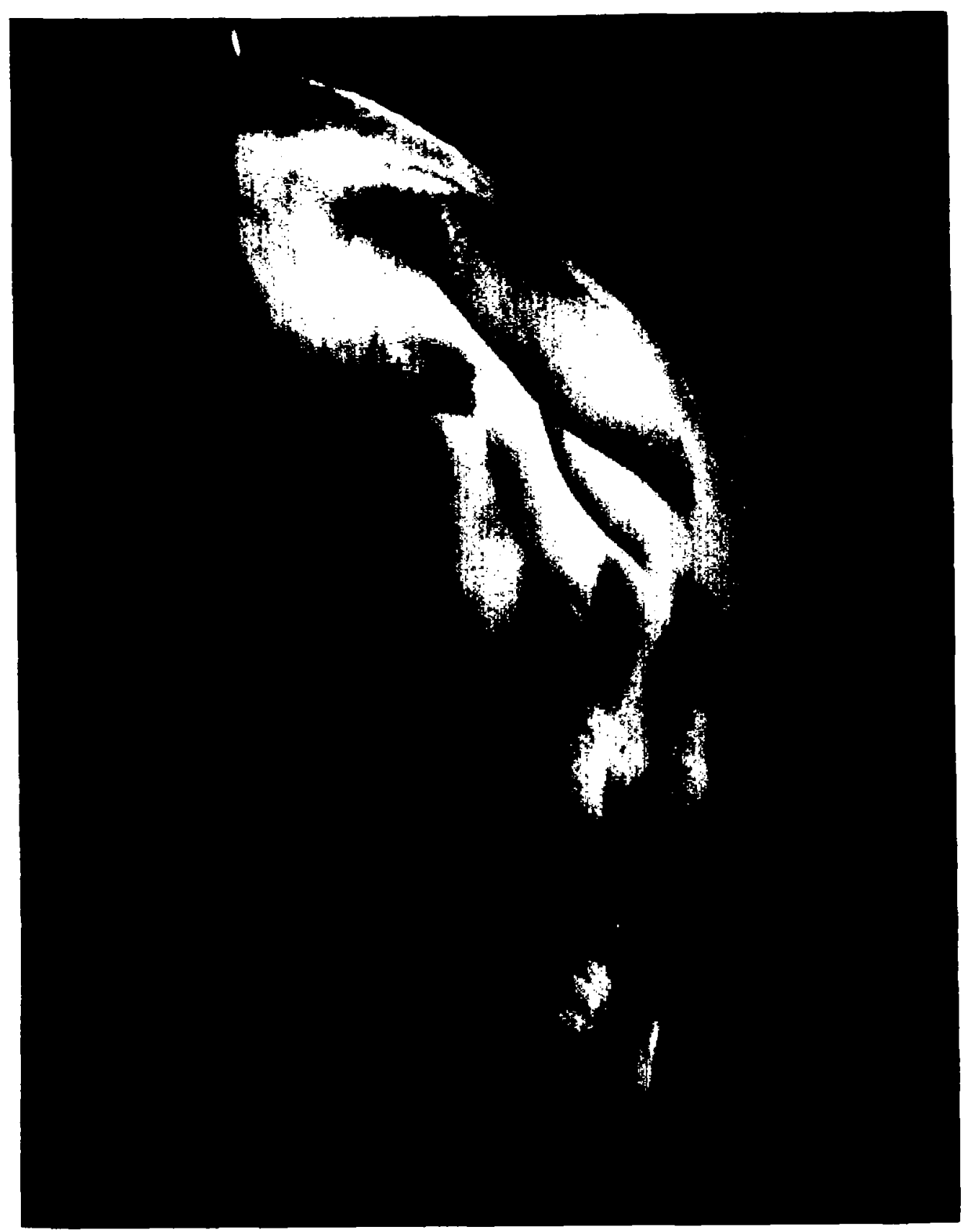

FIG. 1. Perturbations in the development of the solidification front in continuous casting of aluminum alloy. 

The problem as stated has a simple one-dimensional solution in which the moving melt/solid boundary is a plane $y=s_{0}(t)$, and temperatures and stresses depend on $y, t$ only. We shall refer to this as the "zeroth order" solution. However, if a very small spatial perturbation was introduced into the mold temperature or the thermal resistance $R$, the thermomechanical coupling associated with the boundary condition (2) might lead to unstable growth of an associated perturbation in the temperature and stress fields. In particular, we consider the case where the perturbation is spatially sinusoidal and hence the heat flux has the form

$$
Q(x, t)=K \frac{\partial T}{\partial y}(x, 0, t)=Q_{0}(t)+Q_{1}(t) \cos (m x) .
$$

The perturbation is assumed to be sufficiently small for the corresponding twodimensional problem to be solved by linear perturbation of the zeroth order solution. It follows that all the field quantities will also contain perturbations that are sinusoidal in $x$ and the solution for these perturbations will be referred to as the "first order" solution. In particular, the melt/solid boundary will have the sinusoidal form shown in Fig. 2 and defined by

$$
y=s(x, t)=s_{0}(t)+s_{1}(t) \cos (m x),
$$

where $s_{1} \ll s_{0}$ for all $t$. Linearity of the first order problem also requires that the slope of the boundary $\partial s / \partial x$ be small at all times and hence that $m s_{1}(t) \ll 1$.

\section{THE HEAT CONDUCTION PROBLEM}

The temperature is required to satisfy the heat conduction equation:

$$
\nabla^{2} T=\frac{1}{k} \frac{\partial T}{\partial t}
$$

and the boundary conditions:

$$
\begin{aligned}
T(x, s, t) & =T_{\mathrm{m}} \\
K \frac{\partial T}{\partial y}(x, s, t) & =L \rho \frac{\partial s}{\partial t}(x, t),
\end{aligned}
$$

where $\rho$ is the density of the material. The boundary condition (7) defines a heat balance at the moving solid/melt front. Notice that the linearity condition $\partial s / \partial x \ll 1$ permits us to write this condition in terms of $x, y$ rather than in a slightly rotated coordinate system normal to the local front.

In addition, the temperature must satisfy the thermal resistance boundary condition (2). At this stage in the analysis, it is convenient to solve the thermal problem as if the heat flux $Q$ were prescribed, i.e. to use (3) as the additional boundary condition. An additional equation relating $Q$ and $P$ will then be obtained from the thermoelastic analysis, permitting the coupled problem to be solved.

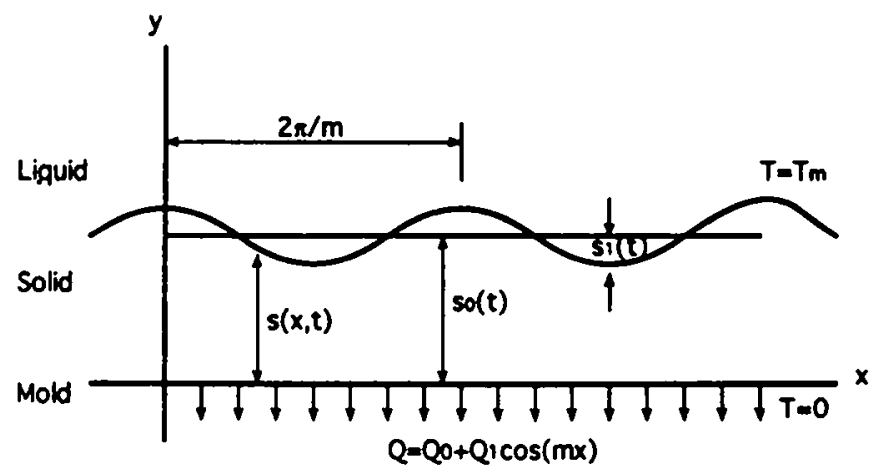

FiG. 2. Geometry of the system. 


\subsection{The perturbation method}

The zeroth and first order problems can be separated by assuming that the temperature can be expressed in the form:

$$
T(x, y, t)=T_{0}(y, t)+T_{1}(y, t) \cos (m x) .
$$

substituting into Eqns (3), (5) - (7) and separating periodic and uniform terms. Thus, Eqn (5) yields

$$
\begin{aligned}
& \frac{i^{2} T_{0}}{i v^{2}}=\frac{1}{k} \quad \frac{i}{i} \\
& \frac{\partial^{2} T_{1}}{\partial y^{2}}-m^{2} T_{1}=\frac{1}{k} \frac{\partial T_{1}}{\partial t} .
\end{aligned}
$$

Since the perturbation is small, we can expand the temperature field in the vicinity of the mean solid/melt interface position, $y=s_{0}(t)$, as a Taylor series. The boundary condition ( 6 ) can be separated into uniform and periodic terms by dropping second order and product terms in the small quantities $T_{1}, s_{1}$ giving the two equations

$$
T_{0}\left(s_{0}, t\right)=T_{\mathrm{m}} ; \quad s_{1}(t) \frac{\mathrm{d} T_{0}}{\hat{r} y}\left(s_{0}, t\right)+T_{1}\left(s_{0}, t\right)=0 .
$$

A similar procedure applied to the boundary condition (7) yields

$$
\begin{aligned}
K \frac{\partial T_{0}}{\partial y}\left(s_{0}, t\right) & =L \rho \frac{\mathrm{d} s_{0}}{\mathrm{~d} t} \\
K\left[\frac{\partial T_{1}}{\partial y}\left(s_{0}, t\right)+s_{1}(t) \frac{\partial^{2} T_{0}}{\partial y^{2}}\left(s_{0}, t\right)\right] & =L \rho \frac{\mathrm{d} s_{1}}{\mathrm{~d} t} .
\end{aligned}
$$

Finally, Eqns (3) and (8) give:

$$
K \frac{\partial T_{0}}{\partial y}(0, t)=Q_{0}(t) ; \quad K \frac{\partial T_{1}}{\partial y}(0, t)=Q_{1}(t) .
$$

The numerical algorithm used to determine the temperature field defined by these equations will be discussed in Section 6 below.

\section{DETERMINATION OF THE THERMOELASTIC STRESS FIELD}

As explained in the Introduction, the solidified material is assumed to be linear elastic and hence the thermoelastic stress field can be determined using the procedure developed by $\mathrm{Li}$ and Barber [8]. Notice, however, that in their case the simplification $\varepsilon=0$ permitted the temperature field to be obtained in closed form, whereas in the present problem it is determined by a numerical algorithm and is therefore defined in discrete form.

\subsection{The zeroth order solution}

The zeroth order quantities define a one-dimensional problem in which the temperatures and hence the stresses depend on $y, t$ only and the shear stress $\sigma_{x y}$ is identically zero. It follows from the equilibrium condition:

$$
\frac{\partial \sigma_{x y_{0}}}{\partial x}+\frac{\partial \sigma_{y y_{0}}}{\partial y}=0
$$

that the component $\sigma_{y y}$ must be independent of $y$ and hence:

$$
\sigma_{y y_{0}}=-p
$$

for all $y, t$, where $p$ is the hydrostatic pressure in the liquid. In particular, this implies that the zeroth order component of the contact pressure distribution $P_{0}(t)$ of Eqn (2) is:

$$
P_{0}(t)=p \text {. }
$$

Notice that these results make no appeal to the elastic constitutive law and are therefore also applicable to the more general case of an elastic/plastic or viscoelastic material. 
The remaining stress component $\sigma_{x x_{0}}$ is determined from the considerations that (i) material is in a state of hydrostatic compression $p$ at the instant of solidification and (ii) thereafter the strain components $\varepsilon_{x x}, \varepsilon_{z z}$, remain unchanged. Application of Hooke's law to these conditions and Eqn (16) then yields the result:

$$
\sigma_{x x_{0}}=-p+\frac{E \alpha}{(1-v)}\left[T_{m}-T_{0}(y, t)\right] .
$$

For an alternative derivation of this result, see Yigit and Barber [10] Section 3.1.

\subsection{The first order solution}

We next consider the thermoelastic problem corresponding to the first order temperature field $T_{1}(y, t) \cos (m x)$. Since the analysis is closely related to that of $\mathrm{Li}$ and Barber [8], only the essential steps are presented in the following derivations, the reader being referred to Ref. [8] for more details.

A suitable particular solution for the stress field can be defined in terms of a thermoelastic displacement potential $\phi$ (see Ref. [11], section 64), where

$$
\phi=f(y, t) \cos (m x) \text {. }
$$

Equation (2) of Ref. [18] then requires that $f$ satisfy the equation

$$
\frac{\partial^{2} f}{\partial y^{2}}(y, t)-m^{2} f(y, t)=\frac{E \alpha}{(1-v)} T_{1}(y, t) \text {. }
$$

Notice that this is essentially an ordinary differential equation for $f$ in which $t$ appears only as a parameter. The first order stress components corresponding to this potential are then obtained by substituting into Eqns (3) of Ref. [8] in the form

$$
\begin{aligned}
\sigma_{x x_{1}}^{P} & =f^{\prime \prime}(y, t) \cos (m x) ; \quad \sigma_{x y_{1}}^{P}=-m f^{\prime}(y, t) \sin (m x) ; \\
\sigma_{y y_{1}}^{P} & =m^{2} f(y, t) \cos (m x),
\end{aligned}
$$

where $(')$ denotes differentiation with respect to $y$.

To satisfy the boundary conditions of the problem, the particular solution must be supplemented by a homogeneous solution which we represent in terms of an Airy stress function $\Phi$. Since the strain rates, but not the strains, are required to be compatible, it follows that the time derivative of $\Phi$ must be biharmonic and hence that the most general function of the appropriate sinusoidal form in $x$ is:

$$
\Phi=\left\{\left[a_{1}(t)+a_{2}(t) y\right] \cosh (m y)+\left[a_{3}(t)+a_{4}(t) y\right] \sinh (m y)+g(y)\right\} \cos (m x),
$$

where the arbitrary time-dependent coefficients $a_{1}, a_{2}, a_{3}, a_{4}$ and the arbitrary time-independent function $g(y)$ are to be determined from the mechanical boundary conditions corresponding to the first order problem.

Using Eqns (1) and (4) of Ref. [8] and Eqns (21) and (22) above, we can construct the complete solution of the first order problem in the form:

$$
\begin{aligned}
\sigma_{x x 1}(x, y, t)= & \left\{\left[a_{1}(t)+a_{2}(t) y+\frac{2 a_{4}(t)}{m}\right] \cosh (m y)\right. \\
& +\left[a_{3}(t)+a_{4}(t) y+\frac{2 a_{2}(t)}{m}\right] \sinh (m y) \\
& \left.+\frac{1}{m^{2}}\left[g^{\prime \prime}(y)-f^{\prime \prime}(y, t)\right]\right\} m^{2} \cos (m x) \\
\sigma_{x y 1}(x, y, t)= & \left\{\left[a_{1}(t)+a_{2}(t) y+\frac{a_{4}(t)}{m}\right] \sinh (m y)\right. \\
& +\left[a_{3}(t)+a_{4}(t) y+\frac{a_{2}(t)}{m}\right] \cosh (m y) \\
& \left.+\frac{1}{m}\left[g^{\prime}(y)-f^{\prime}(y, t)\right]\right\} m^{2} \sin (m x)
\end{aligned}
$$




$$
\begin{aligned}
\sigma_{y y 1}(x, y, t)=\{ & -\left[a_{1}(t)+a_{2}(t) y\right] \cosh (m y) \\
& \left.-\left[a_{3}(t)+a_{4}(t) y\right] \sinh (m y)-g(y)+f(y, t)\right\} m^{2} \cos (m x) .
\end{aligned}
$$

Also, using the elastic constitutive relation for plane strain to determine the strain components and hence solve for the displacements, we obtain:

$$
\begin{aligned}
\dot{u}_{y 1}(x, y, t)= & -\frac{1+v}{E}\left\{-\frac{1}{m}\left[\dot{f}^{\prime}(y, t)\right]+\left[\dot{a}_{1}(t)+\dot{a}_{2}(t) y-\frac{(1-2 v)}{m} \dot{a}_{4}(t)\right] \sinh (m y)\right. \\
& +\left[\dot{a}_{3}(t)+\dot{a}_{4}(t) y-\frac{1}{m} \frac{2 v}{m} \dot{a}_{2}(t) \mid \cosh (m y)\right\} m \cos (m x),
\end{aligned}
$$

where $\left({ }^{\circ}\right)$ denotes differentiation with respect to $t$.

We now consider the boundary conditions corresponding to the first order problem. Since the perturbation on the stress field is small, we can expand the stress field in the vicinity of the mean solid/liquid interface position, $y=s_{0}(t)$, as a Taylor series. Then the first boundary condition in Eqn (20) of Ref. [8] can be written, dropping second, higher and product terms in small quantities, $\sigma_{1}, s_{1}$, as:

$$
\sigma_{x x y}\left(s_{0}, t\right)+\sigma_{x \times 0}^{\prime}\left(s_{0} . t\right) s_{1}(t) \cos (m x)+\sigma_{x x 1}\left(x, s_{0}, t\right)=-p .
$$

Separating periodic and uniform terms and using Eqn (18), we obtain the boundary condition for $\sigma_{\lambda \times 1}$ at $y=s_{0}$, i.e.

$$
\sigma_{x \times 1}\left(x, s_{0}(t), t\right)=\frac{E \alpha}{(1-v)} T_{0}^{\prime}\left(s_{0}, t\right) s_{1}(t) \cos (m x) .
$$

Applying the same procedure to the remaining boundary conditions in Eqn (20) of Ref. [8]. we obtain:

$$
\sigma_{x y 1}\left(x, s_{0}, t\right)=0 ; \quad \sigma_{y y 1}\left(x, s_{0}, t\right)=0 .
$$

Also, from Eqns (21) and (22) of Ref. [8], we have:

$$
\sigma_{x, 1}(x, 0, t)=0 ; \quad \dot{u}_{y 1}(x, 0, t)=0 .
$$

Applying the boundary conditions (30), using Eqns (24) and (26), we can determine the time derivatives of coefficients $a_{2}(t)$ and $a_{3}(t)$. i.c.

$$
\dot{a}_{2}(t)=0 ; \quad \dot{a}_{3}(t)=\frac{1}{m} \dot{f}^{\prime}(0, t)
$$

We can therefore write:

$$
a_{2}(t)=0 ; \quad a_{3}(t)=\frac{1}{m} f^{\prime}(0, t)
$$

without loss of generality, since constants of integration will lead to time-independent terms in $\Phi$ which can be subsumed under $g(y)$.

Substituting for the stress components from Eqns (23)-(25) into the remaining boundary conditions (28) and (29). we obtain the equations

$$
\begin{aligned}
& a_{1}(t) \cosh \left(m s_{0}(t)\right)+a_{4}(t)\left[\frac{2}{m} \cosh \left(m s_{0}(t)\right)+s_{0}(t) \sinh \left(m s_{0}(t)\right)\right] \\
& +\frac{1}{m^{2}}\left[g^{\prime \prime}\left(s_{0}(t)\right)-f^{\prime \prime}\left(s_{0}(t), t\right)\right]=\frac{E \alpha}{\left(1-\frac{\alpha}{v) m^{2}} T_{0}^{\prime}\left(s_{0}(t), t\right) s_{1}(t)\right.} \\
& \quad-\frac{1}{m} \sinh \left(m s_{0}(t)\right) f^{\prime}(0, t) \\
& a_{1}(t) \sinh \left(m s_{0}(t)\right)+a_{4}(t)\left[\frac{1}{m} \sinh \left(m s_{0}(t)\right)+s_{0}(t) \cosh \left(m s_{0}(t)\right)\right] \\
& +\frac{1}{m} g^{\prime}\left(s_{0}(t)\right)=\frac{1}{m} f^{\prime}\left(s_{0}(t), t\right)-\frac{1}{m} f^{\prime}(0, t) \cosh \left(m s_{0}(t)\right)
\end{aligned}
$$




$$
\begin{aligned}
& a_{1}(t) \cosh \left(m s_{0}(t)\right)+a_{4}(t) s_{0}(t) \sinh \left(m s_{0}(t)\right)+g\left(s_{0}(t)\right) \\
& =f\left(s_{0}(t), t\right)-\frac{\sinh \left(m s_{0}(t)\right)}{m} f^{\prime}(0, t),
\end{aligned}
$$

where we have used Eqn (32) to eliminate $a_{2}(t)$ and $a_{3}(t)$. These three equations must be satisfied for all values of $t$ and hence we can use them to eliminate $a_{1}(t)$ and $a_{4}(t)$. Defining $\omega=\tanh \left(m s_{0}(t)\right)$, we obtain:

$$
\begin{aligned}
\frac{1}{2 m^{2}}[ & \left.m s_{0}(t) \omega^{2}-\omega-m s_{0}(t)\right] g^{\prime \prime}\left(s_{0}(t)\right)+\frac{1}{m} g^{\prime}\left(s_{0}(t)\right)-\frac{1}{2}\left[m s_{0}(t) \omega^{2}\right. \\
& \left.+\omega-m s_{0}(t)\right] g\left(s_{0}(t)\right)=\frac{1}{2 m^{2}}\left[m s_{0}(t) \omega^{2}-\omega-m s_{0}(t)\right] f^{\prime \prime}\left(s_{0}(t), t\right) \\
& -\frac{1}{2}\left[m s_{0}(t) \omega^{2}+\omega-m s_{0}(t)\right] f\left(s_{0}(t), t\right)+\frac{1}{m} f^{\prime}\left(s_{0}(t), t\right)-\frac{f^{\prime}(0, t)}{m \cosh \left(m s_{0}(t)\right)} \\
& +\frac{1}{2}\left[m s_{0}(t) \omega^{2}-\omega-m s_{0}(t)\right] \frac{E \alpha s_{1}(t)}{(1-v) m^{2}} T_{0}^{\prime}\left(s_{0}(t), t\right),
\end{aligned}
$$

which serves to determine the unknown residual stress function $g(y)$. Once $g(y)$ is known, we can recover $a_{1}(t)$ and $a_{4}(t)$ by solving Eqns (33) and (35), with the result:

$$
\begin{aligned}
a_{1}(t)= & \frac{1}{\cosh \left(m s_{0}(t)\right)}\left\{f\left(s_{0}(t), t\right)-\frac{\sinh \left(m s_{0}(t)\right)}{m} f^{\prime}(0, t)-g\left(s_{0}(t)\right)\right. \\
& -\frac{m s_{0}(t) \omega}{2}\left[\frac{E \alpha}{(1-v) m^{2}} s_{1}(t) T_{0}^{\prime}\left(s_{0}(t), t\right)+\frac{1}{m^{2}}\left[f^{\prime \prime}\left(s_{0}(t), t\right)-g^{\prime \prime}\left(s_{0}(t)\right)\right]\right. \\
& \left.\left.-f\left(s_{0}(t), t\right)+g\left(s_{0}(t)\right)\right]\right\} \\
a_{4}(t)= & \frac{m}{2 \cosh \left(m s_{0}(t)\right)}\left\{\frac{E \alpha}{(1-v) m^{2}} s_{1}(t) T_{0}^{\prime}\left(s_{0}(t), t\right)\right. \\
& \left.+\frac{1}{m^{2}}\left[f^{\prime \prime}\left(s_{0}(t), t\right)-g^{\prime \prime}\left(s_{0}(t)\right)\right]-\left[f\left(s_{0}(t), t\right)-g\left(s_{0}(t)\right)\right]\right\} .
\end{aligned}
$$

Finally, we determine the perturbation in contact pressure $P_{1}(t) \cos (m x)$ at the casting/mold interface, from Eqns (25) of Ref. [8] and Eqns (25) and (32) as:

$$
P_{1}(t)=m^{2}\left[a_{1}(t)-f(0, t)\right],
$$

where we have imposed the arbitrary condition $g(0)=0$, since the free constants in the solution of Eqn (36) can be assigned to satisfy this condition.

\section{DIMENSIONLESS PRESENTATION}

Before proceeding to the solution for the coupled problem, it is convenient to define the new dimensionless parameters.

$$
\begin{gathered}
Y=m y ; \quad \beta=m^{2} \frac{K T_{\mathrm{m}}}{\rho L} t ; \quad S(\beta)=m s(t) ; \\
\bar{T}(Y, \beta)=\frac{T(y, t)}{T_{\mathrm{m}}} ; \quad \bar{Q}=\frac{Q}{m K T_{\mathrm{m}}} ; \quad \bar{P}(\beta)=\frac{1-v}{E \alpha T_{\mathrm{m}}} P(t) ; \\
F(Y, \beta)=\frac{m^{2}(1-v)}{E \alpha T_{\mathrm{m}}} f(y, t) ; \quad G(Y)=\frac{m^{2}(1-v)}{E \alpha T_{\mathrm{m}}} g(y) ; \\
\bar{R}_{0}=m K R_{0} ; \quad \bar{R}^{\prime}=\frac{E \alpha T_{\mathrm{m}} m K}{1-v} R^{\prime}\left(P_{0}\right) .
\end{gathered}
$$


The governing Eqns (9) and $(10)$ for $\bar{T}_{0}(Y, \beta)$ and $\bar{T}_{1}(Y, \beta)$ then become:

$$
\frac{\hat{c}^{2} \bar{T}_{0}}{\partial Y^{2}}=\varepsilon \frac{\partial \bar{T}_{0}}{\partial \beta}: \frac{\hat{c}^{2} \bar{T}_{1}}{\partial Y^{2}}-\bar{T}_{1}=\varepsilon \frac{\partial \bar{T}_{1}}{\partial \beta} .
$$

The boundary conditions (11a), (12) and (14a) corresponding to the zeroth order temperature field, $\bar{T}_{0}(Y, \beta)$ become:

$$
\bar{T}_{0}\left(S_{0}, \beta\right)=1 ; \quad \frac{\partial \bar{T}_{0}\left(S_{0}, \beta\right)}{\hat{c} Y}=\frac{\mathrm{d} S_{0}(\beta)}{\mathrm{d} \beta}: \quad \frac{\partial \bar{T}_{0}(0, \beta)}{\partial Y}=\bar{Q}_{0}(\beta),
$$

and the boundary conditions (11b), (13) and (14b) corresponding to the first order temperature field $\bar{T}_{1}(Y, \beta)$ can be written as:

$$
\begin{gathered}
S_{1}(\beta) \frac{\partial \bar{T}_{0}\left(S_{0}, \beta\right)}{\partial Y}+\bar{T}_{1}\left(S_{0}, \beta\right)=0 \\
\frac{\partial \bar{T}_{1}\left(S_{0}, \beta\right)}{\partial Y}+S_{1}(\beta) \frac{\partial^{2} \bar{T}_{0}\left(S_{0}, \beta\right)}{\partial Y^{2}}=\frac{\mathrm{d} S_{1}(\beta)}{\mathrm{d} \beta} \\
\frac{\partial \bar{T}_{1}(0, \beta)}{\partial Y}=\bar{Q}_{1}(\beta) .
\end{gathered}
$$

Thus, the heat conduction problem is reduced to the determination of two pairs of functions $\bar{T}_{0}(Y, \beta), S_{0}(\beta)$ and $\bar{T}_{1}(Y, \beta), S_{1}(\beta)$ in Eqns $(44)$ which satisfy the boundary conditions $(45)$ and (46), respectively. These equations would completely define the temperature field if the heat flux $Q(x, t)$ of Eqn (3) were prescribed, as in Ref. [10], but, in the present problem, the heat flux is related to the temperature and the contact pressure through Eqn (2), thus coupling the thermal and mechanical problems.

However, the zeroth order problem does not exhibit this coupling because the contact pressure is constant [see Eqn (17)]. We can therefore write:

$$
\bar{Q}_{0}=\frac{\bar{T}_{0}(0, \beta)}{\bar{R}_{0}}
$$

from Eqns (2), (41) and (43), where $\bar{R}_{0}$ is the dimensionless contact resistance associated with the (constant) zeroth order contact pressure, $P_{0}$. With this additional condition, the zeroth order temperature field is completely defined. After it is found, the corresponding stress field can be determined from Eqn (18).

By contrast, the first order problem is fully coupled and we must use the stress analysis of Section 4.2 to obtain a relation between $Q_{1}(t)$ and $P_{1}(t)$ which, in combination with the perturbed form of Eqn (2), provides the additional condition required for the solution. The procedure here is to solve Eqn (20) for $f(y, t)$ and Eqn (36) for $g(y)$, after which we can find $a_{1}(t)$ from Eqn (37) and hence $P_{1}(t)$ from Eqn (39). The dimensionless form of these equations is:

$$
\begin{gathered}
\frac{\partial^{2} F(Y, \beta)}{\partial Y^{2}}-F(Y, \beta)=\bar{T}_{1}(Y, \beta) \\
\frac{1}{2}\left[\bar{\omega}^{2} Y-\bar{\omega}-Y\right] G^{\prime \prime}(Y)+G^{\prime}(Y)+\frac{1}{2}\left[-\bar{o}^{2} Y-\bar{\omega}+Y\right] G(Y)= \\
-\frac{S_{1}\left(\beta_{0}(Y)\right)}{2}\left[\bar{\omega}+Y-\bar{\omega}^{2} Y\right] \bar{T}_{0}^{\prime}\left(Y, \beta_{0}(Y)\right)+\frac{1}{2}\left[Y-\bar{\omega}-\bar{\omega}^{2} Y\right] F\left(Y, \beta_{0}(Y)\right) \\
+\left[F^{\prime}\left(Y, \beta_{0}(Y)\right)-\frac{F^{\prime}\left(0, \beta_{0}(Y)\right)}{\cosh (Y)}\right]+\frac{1}{2}\left[\bar{\omega}^{2} Y-\bar{\omega}-Y\right] F^{\prime \prime}\left(Y, \beta_{0}(Y)\right) \\
\bar{a}_{1}(\beta)=\frac{1}{\cosh \left(S_{0}\right)}\left\{F\left(S_{0}, \beta\right)-\sinh \left(S_{0}\right) F^{\prime}(0, \beta) G\left(S_{0}\right)\right. \\
\left.\quad-\frac{\bar{\omega} S_{0}}{2}\left[S_{1}(\beta) \bar{T}_{0}^{\prime}\left(S_{0}, \beta\right)+F^{\prime \prime}\left(S_{0}, \beta\right)-G^{\prime \prime}\left(S_{0}\right)-F\left(S_{0}, \beta\right)+G\left(S_{0}\right)\right]\right\}
\end{gathered}
$$




$$
\bar{P}_{1}(\beta)=\bar{a}_{1}(\beta)-F(0, \beta) .
$$

Finally, we use the technique developed in Refs $[12,13]$ to obtain a relation between the small perturbations $\Delta Q=Q_{1}(t) \cos (m x), \Delta T=T_{1}(0, t) \cos (m x), \Delta P=P_{1}(t) \cos (m x)$ from the zeroth order values $Q_{0}, T_{0}, P_{0}$. Differentiating Eqn (2), we obtain:

$$
\frac{\Delta Q}{Q_{0}}=\frac{\Delta T}{T_{0}}-\frac{R^{\prime}\left(P_{0}\right) \Delta P}{R_{0}},
$$

where $R^{\prime}=\mathrm{d} R / \mathrm{d} P$ defines the sensitivity of the thermal contact resistance to small changes of pressure about the mean value $P_{0}$.

Each of the perturbed quantities changes with $x$ only through the multiplier $\cos (m x)$ and hence Eqn (52) in dimensionless form reduces to:

$$
\frac{\bar{Q}_{1}(\beta)}{\bar{Q}_{0}(\beta)}=\frac{\vec{T}_{1}(0, \beta)}{\bar{T}_{0}(0, \beta)}-\frac{\bar{R}^{\prime}\left(\bar{P}_{0}\right) \bar{P}_{1}(\beta)}{\bar{R}_{0}} .
$$

\section{NUMERICAL ALGORITHM}

Both zeroth order and first order problems require numerical solution, for which we use the algorithm developed in Ref. [10]. The zeroth order solid phase $0<Y<S_{0}(t)$, is divided into a fixed number of elements $N$, so the space step size, $\delta=S_{0} / N$, increases with time due to the growth in $S_{0}(t)$. This permits the last node to be identified with the zeroth-order melt/solid front at all times, but implies that the node locations move in time, necessitating the inclusion of convective terms in the updating algorithm for temperature. Thus, for example, the instantaneous zeroth order temperature field is represented by the temperatures at the $N+1$ points $Y=i \delta, i=0, N$. The increase of the layer thickness $S_{0}$ during the next time increment $\tau$ is determined from the finite difference form of Eqn (45b) after which the temperatures at the interior nodes $i=1,(N-1)$ are updated using the heat conduction Eqn (44a) including convective terms. The temperature at node $N$ remains at 1 for all times in view of Eqn (45a) and that at node 0 is updated by first determining the instantaneous heat flux from Eqn (45c), which determines the first difference in the first element.

Essentially, the same procedure is used to determine the evolution of the first order temperature field, using Eqns (44b) and (46), except that the heat flux $\bar{Q}_{1}$ must be determined from Eqn (53), which necessitates the solution of the thermoelastic problem for $\bar{P}_{1}$, using Eqns $(48)-(51)$. If the time increment $\tau$ is sufficiently small, the thermal and thermoelastic updating algorithms can be performed sequentially and hence explicitly.

The choice of an appropriate value for $\tau$ is motivated by the desire for computational efficiency, whilst retaining acceptable numerical convergence and stability. Extensive investigations were made into the effect of both space and time discretization to ensure that the final results are reliable. All the results presented below were obtained using 100 elements. With the explicit scheme used here, the maximum time step for stability is proportional to $\delta^{2} / k$ and hence the stability requirement generally places the most severe restrictions on $\tau$ when good spatial accuracy is desired, necessitating small values of $\delta$. However, $S_{0}$ and hence $\delta$ increase during the process, permitting the time step to be increased as the system evolves, without loss of stability.

\subsection{Initial conditions}

With the algorithm described above, it is clearly not possible to start from the instant of first solidification, since at $S_{0}=0$, all the nodes would coincide. Instead, we need to use an asymptotic solution of the problem at small times to provide a suitable initial condition for the numerical algorithm at finite $\beta$. Fortunately, when the solidified layer is very thin, the temperature drop across it is small compared with that across the thermal contact resistance. It follows that the effective Stefan number is small and to the first order, the heat flux $Q_{0}$ has the constant value $T_{\mathrm{m}} / R_{0}$.

The solution of $\mathrm{Li}$ and Barber [8] therefore constitutes an appropriate asymptotic solution of the more general problem at small times and can be used as an initial condition for the numerical algorithm. Since $S_{0}$ and hence $\delta$ is small at small times, there are 
computational advantages in starting the algorithm with as large a value of $\beta$ as possible. Suitable starting times were determined by imposing the condition that the evolution predicted by the algorithm follow the asymptotic solution for a significant period of time.

\section{RESULTS}

Figure 3 shows the effect of Stefan number on the growth of the perturbation $S_{1}(\beta)$ in the solidification front for $\bar{R}^{\prime}=-100$ and $\bar{R}_{0}=0.3$. Notice that $\bar{R}^{\prime}$ will generally be negative because the thermal contact resistance falls with increasing contact pressure.

As in the idealized problem of $\mathrm{Li}$ and Barber [8], an initial perturbation must be given to the system, corresponding to a non-zero value of $S_{1}^{\prime}(0)$. The first order equations are linear (in contrast to the non-linear zeroth order process), and hence all the perturbed quantities are proportional to this initial perturbation. We therefore present the ratio $S_{1}(\beta) / S_{1}^{\prime}(0)$ in Fig. 3. Results for any desired value of $S_{1}^{\prime}(0)$ can be found from this figure by linear scaling.

The results confirm the hypothesis of Yigit and Barber [10] that the thermal capacity of the material has a significant stabilizing effect on the growth of the instability, the perturbations recorded for $\varepsilon=1$ being substantially smaller than those for $\varepsilon=0$. Thus, it is essential to include thermal capacity effects in any realistic analysis of contact thermoelastic stability in solidification of metals.

At larger values of time $\beta$, the perturbation in $S_{1}$ reaches a maximum and then decays as shown in Fig. 4. The maximum reached is lower for larger $\varepsilon$ and is reached at a later time.

For given mold temperature and mean thermal contact resistance, increasing $\delta$, i.e. increasing the thermal capacity of the solidifying material, also slows down the progress of the mean solidified front $S_{0}$. We therefore replot the results of Fig. 4 against $S_{0}$ in Fig. 5. The results show that the maximum value of $S_{1}$ occurs at approximately $S_{0}=3$ for all $\varepsilon$. Recalling that $S_{0}=m s_{0}$ and that the wavelength of the perturbation in the $x$-direction is $2 \pi / m$, we conclude that the maximum perturbation in the wavefront occurs when the mean solidified layer thickness $s_{0}$ is approximately half the wavelength of the perturbation.

A simple physical explanation of this result is provided by the fact that the term $T_{1} \cos (m x)$ associated with an imposed perturbation at $y=0$ decays with $\exp (m y)$. Thus. when the dimensionless mean layer thickness approaches the wavelength, there is relatively weak coupling between the perturbations at the mold/solid interface and the solidification front. Since this coupling is the source of the unstable behaviour, stabilization then occurs.

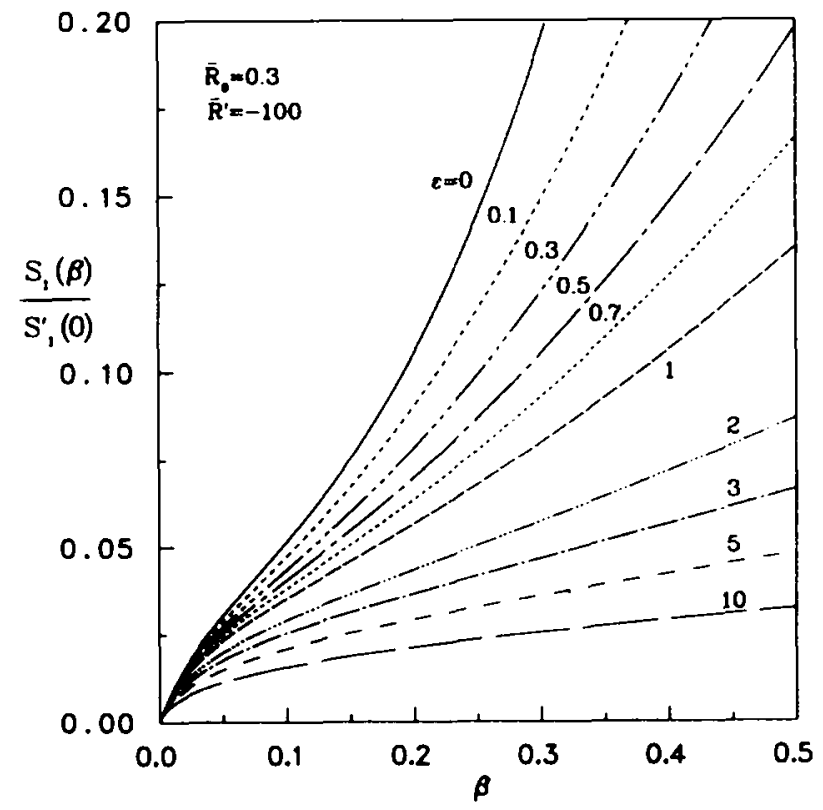

FIG. 3. Normalized perturbation in solidification front as a function of time for $\bar{R}^{\prime}=-100$. $\tilde{R}_{0}=0.3$ and various values of $\varepsilon$. 


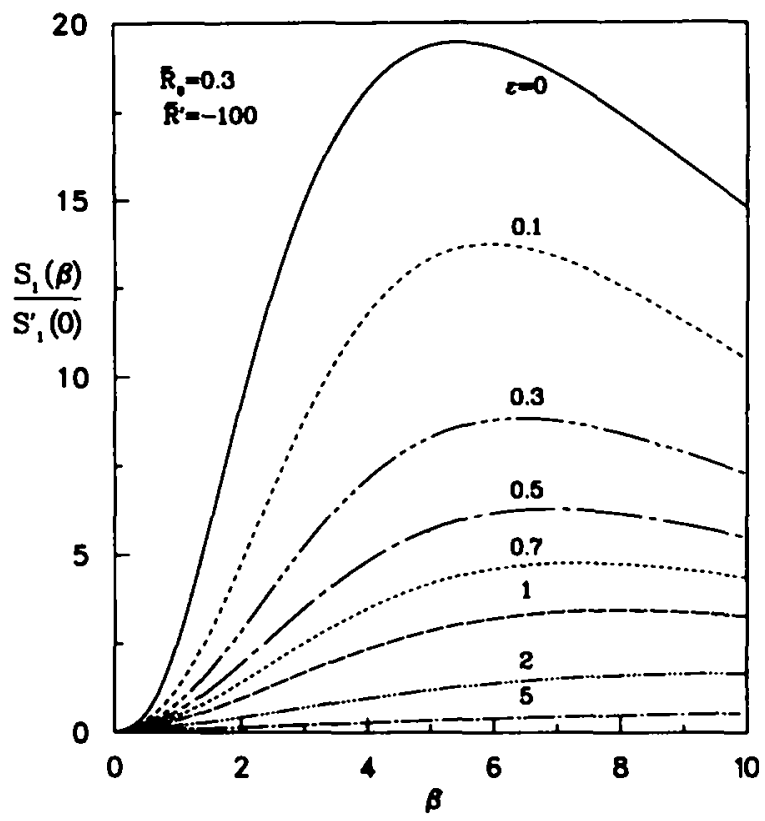

FiG. 4. Normalized perturbation in solidification front at larger values of time for $\bar{R}^{\prime}=-100$, $\hat{R}_{0}=0.3$.

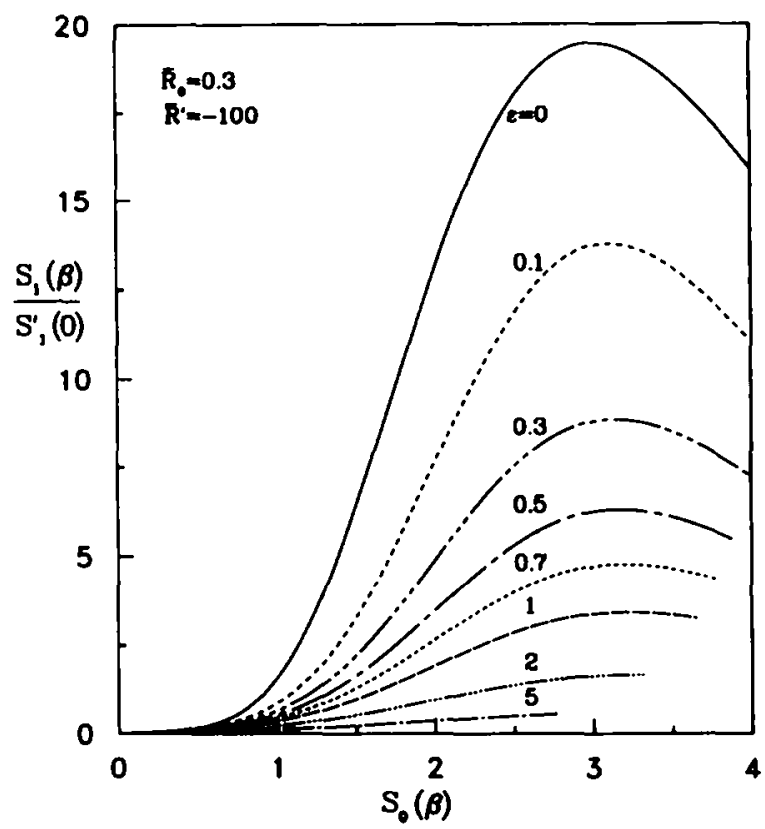

F1G. S. Normalized perturbation in solidification front as a function of $S_{0}(\beta)$ for $\bar{R}^{\prime}=-100$, $\bar{R}_{0}=0.3$ and various values of $\varepsilon$.

Results for $\varepsilon=0$ are provided in the above figures for comparison. These results were not transcribed from the analysis of $\mathrm{Li}$ and Barber [8], since in that paper, an additional assumption was made that $S_{0} \ll 1$ in order to justify the approximation of the first order temperature field by a linear function. It is in fact possible to rework the analysis of $\mathrm{Li}$ and Barber without making this approximation, the essential steps of this derivation being given in the Appendix, and this method was used to develop the curves for $\varepsilon=0$ in the present paper. The results were also used to test the performance of the numerical algorithm at small $\varepsilon$, excellent agreement being obtained between the two solutions. 
The parameter $\bar{R}^{\prime}$ describes the pressure-sensitivity of the thermal contact resistance and is therefore the driving force for the mechanism under investigation. We should thereforc expect that smaller (negative) values of $\bar{R}^{\prime}$ would result in more "stable" behavior. This is supported by the results shown in Fig. 6 for $\bar{R}_{0}=0.3, \varepsilon=0$ and a range of values of $\ddot{k}$. Notice that we had to use a logarithmic scale to fit all the curves on one figure, demonstrating that the pressure-sensitivity of thermal contact resistance has a major effect on the response to a given initial perturbation. In this figure, we also present the curve for $\bar{R}=0$ corresponding to the case where there is no thermomechanical coupling and the intial perturbation remains unchanged by the subsequent process. This provides a benchmark for the other curves and indicates that a value of $\bar{R}^{\prime}<-20$ is needed for the proposed mechanism to produce significant thermomechanically driven growth of the perturbation.

Results were also obtained for many other values of the parameters. It was found that increasing Stefan number always reduces the rate of growth of the perturbation, but the effect is most noticeable for cases which in the zero Stefan number limit appear most "unstable". A convenient measure of the stability of the process is provided by the maximum value of the ratio $S_{1}(\beta) S_{1}^{\prime}(0)$, i.e. the height of the peak in the curves of Figs 46 . We present this value for various Stefan numbers as a function of $\bar{R}^{\prime}$ for $R_{0}=0.3$ in Fig. ? and as a function of $\bar{R}_{0}$ for $\bar{R}^{\prime}=\cdots 100$ in Fig. 8 . Figure 7 shows that the growth of the perturbation is always increased by a greater negative value of $\bar{R}^{\prime}$. confirming the trend shown for zero Stefan number in Fig. 6. Figure 8 shows that reducing $\bar{R}_{0}$ generally conduces to instability, but only if the Stefan number is relatively small. A major consequence of increasing $\bar{R}_{0}$ is to reduce the cooling rate $\bar{Q}_{0}$ [see Eqn (47)] and hence slow down the zeroth order process. We thus conclude that the effect of Stefan number is most important at high cooling rates, where the greatest growth of the perturbation is observed.

\section{$x$ CONCLLISIONS}

The results document the effect of Stefan number on the growth of a perturbation in the nominally plane solidification front due to thermomechanical coupling associated with a pressure-dependent thermal contact resistance. The Stefan number is a measure of the influence of thermal capacity on the solution and previous analyses have generally been restricted to the case of zero Stefan number, corresponding to a solidifying material of zero

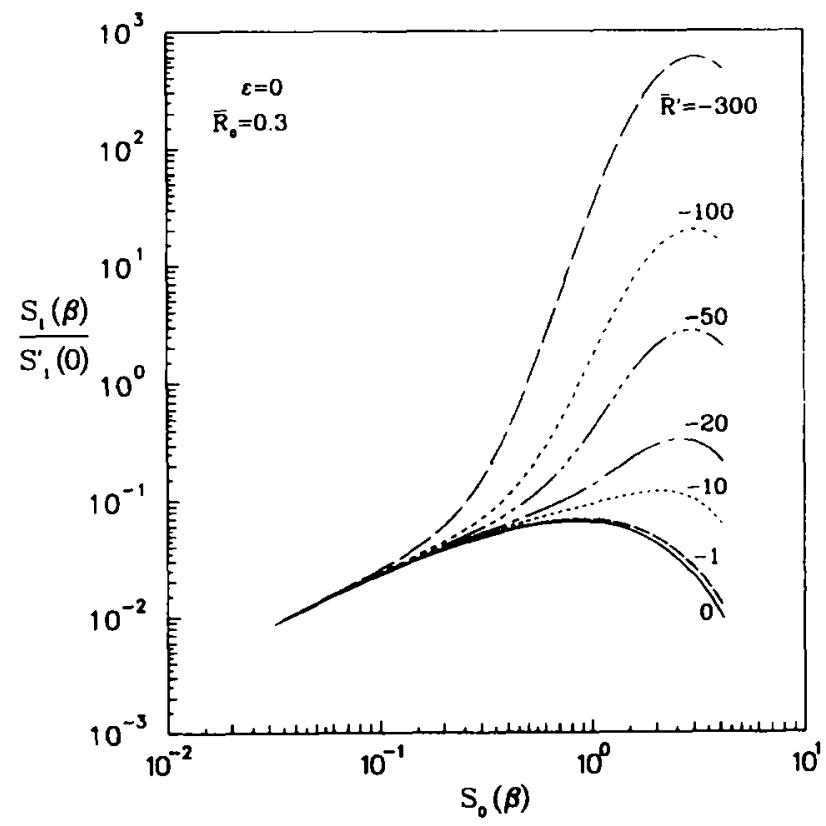

FIG. 6. Normalized perturbation in solidification front as a function of $S_{0}(\beta)$ for $\bar{R}_{0}=0.3, \varepsilon=0$ and a range of values of $\vec{R}^{\prime}$. 


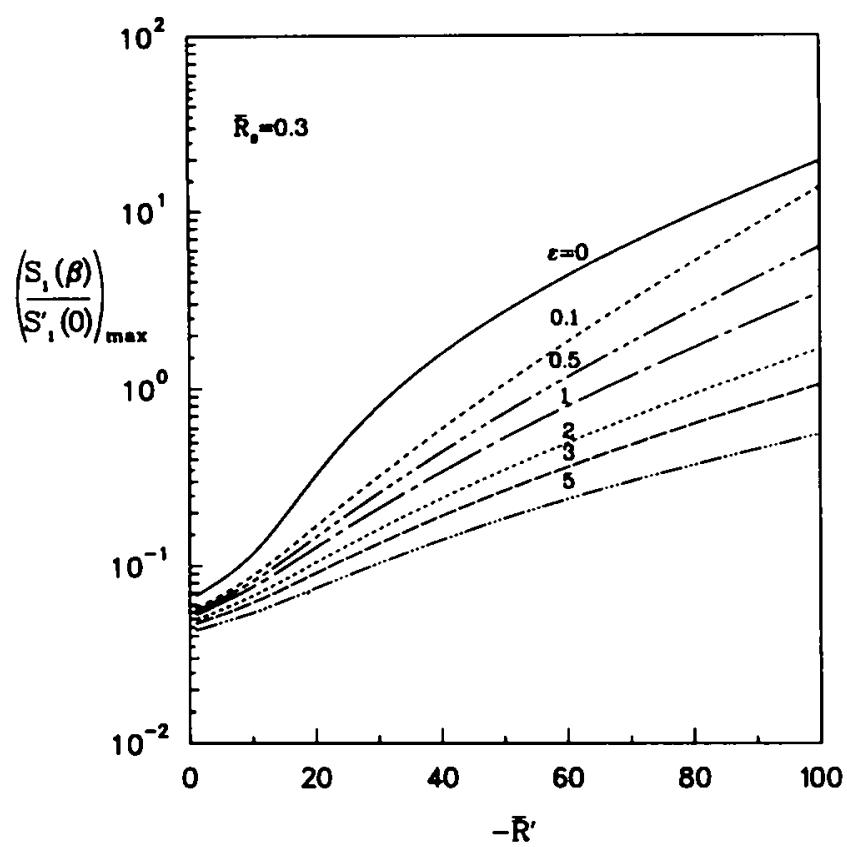

FIG. 7. Maximum normalized perturbation in solidification front as a function of $-\bar{R}^{\prime}$ for $\bar{R}_{0}=0.3$ and various values of $\varepsilon$.

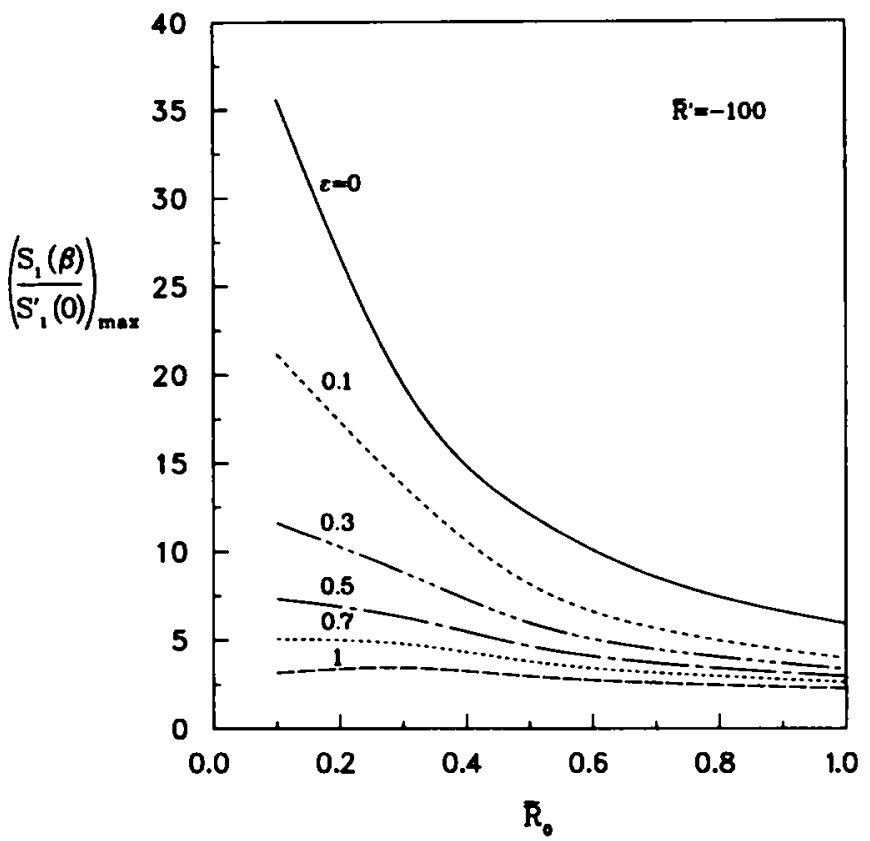

FIG. 8. Maximum normalized perturbation in solidification front as a function of $\bar{R}_{0}$ for $\bar{R}^{\prime}=-100$ and various values of $\varepsilon$.

thermal capacity. In addition, we remove the restriction to small times of the zero Stefan number analysis of $\mathrm{Li}$ and Barber [8].

In all cases, the initial sinusoidal perturbation grows to a maximum amplitude in the solidification front and then decays, the maximum being reached when the mean solidified layer thickness is about half the wavelength of the perturbation.

In general, increasing Stefan number has a stabilizing effect on the process. This effect is most noticeable in cases where the zero Stefan number approximation predicts substantial 
growth of an initial perturbation, e.g. where the thermal contact resistance is very sensitive to pressure.

The inclusion of thermal capacity in the heat conduction solution necessitates a numerical solution of the problem, in contrast to the essentially analytical methods used in previous papers. However, since the growth of the perturbation is linear, the two-dimensional stability problem is reduced to two one-dimensional numerical problems which can be solved sequentially. This represents a considerable saving in computational time, which therefore permits very good accuracy and convergence to be achieved in the numerical solution.

In principle, the same process can be applied to more realistic representations of the problem involving, e.g. a nonlinear constitutive law in the solidified material and or temperature dependent material properties. Additional nonlinearity would then be introduced into the zeroth order thermal and mechanical problems, but the changes during each time step would need to be sufficiently small to permit an explicit or at worst a very convergent iterative solution of the resulting equations. The more complex first order problem would then be governed by linear equations as long as the perturbation is small. These equations would have spatially variable coefficients, determined from the mean local conditions from the zeroth order solution. This is the subject of an ongoing investigation. The process has some similarity with the well-known procedure for determining the effect of small strain perturbations on simple large deformation states (see, e.g. Ref. [14]).

Acknowledgements - The authors are pleased to acknowledge support from the National Science Foundation under contract number MSS-9200762.

\section{REFERENCES}

1. H. Fredriksson and M. Thegerstrom, On the formation of an air gap between the mold and the strand in continuous casting. Scand. Journal of Metallurgy 8,232 (1979).

2. S. N. SINGH and K. E. BLAZEK, Heat transfer and skin formation in a continuous-casting mold as a function of steel carbon content. Journal of Metals 17 (1974).

3. V. Y. NEYMARK, Influence of inoculants and crystallization conditions on the quality of the skin of the ingot, in Transactions of the First All-Union Conference on Continuous Steel Casting, pp. 222-240. Moscow (1955).

4. P. J. WraY, Nonuniform growth of a plate solidifying on a chilled surface. in Proceedings of the AIME Conference, Presentation notes. Atlanta (1977).

5. P. J. WraY, Geometric features of chill-cast structures. Metallurgical Transactions. 12B, 167 (1981).

6. D. L. W. Collins, A new explanation of the surface structures of D.C. ingots. Mettalurgia 76, 137 (1967).

7. O. Richmond, L. G. Hector Jr and J. M. Fridy, Growth instability during nonuniform directional solidification of pure metals. ASME Journal of Applied Mechanics 57, 529 (1990)

8. NAI-YI LI and J. R. BARBER, Thermoelastic instability in planar solidification. International Journal of Mechanical Sciences 33, 945 (1991).

9. H. S. Carslaw and J. C. Jakger. Conduction of Heat in Solids 2nd Edn. Oxford University Press, London. England (1956)

10. F. YIGIT and J. R. BARBER, Effect of thermal capacity on the development of sinusoidal perturbation during the unidirectional solidification of pure metals, in Micro/Macro Scale Phenomeno in Solidification. ASME, HTD-Vol. 218/AMD-Vol. 139, pp. 141-150 (1992).

11. H. M. Westergaard, Theory of Elasticity and Plasticity Art. 64. Dover, New York (1964).

12. J. R. BARBER, J. DUNDURS and M. COMNINOU. Stability considerations in thermoelastic contact. Journal of Applied Mechanics 47, 871 (1980).

13. R. ZhANG and J. R. BARBER, Effects of material propertics on the stability of static thermoelastic contact. Journal of Applied Mechanics 57, 365 (1990).

14. A. E. Green and W. Zerna, in Theoretical Elasticity, Chap. 4. Clarendon Press, Oxford (1954).

\section{APPENDIX: SOLUTION FOR ZERO STEFAN NUMBER}

Results for the corresponding problem with zero Stefan number were presented by Li and Barber [8]. However. they assumed that the temperature $\bar{T}(Y, \beta)$ varied linearly with $Y$, which effectively restricts their analysis to the early stage of the process during which the thickness of the solidified layer is small in comparison with the wavelength of the perturbation, i.e. $S_{0}(\beta) \ll 1$. The solution is readily obtained without this restriction.

The zeroth order solution

Equation (44a) does yield a linear solution for $\bar{T}_{0}$ in the limit $\varepsilon=0$, so $\mathrm{Li}$ and Barber's solution is exact for the zeroth order problem. The corresponding contact resistance $\bar{R}_{0}$ is constant and the solution is defined by

$$
\text { (1) }:(i) \cdot \cdots \cdot \dot{R}_{1}
$$




$$
\begin{aligned}
\bar{T}_{0}(Y, \beta) & =\frac{Y+\bar{R}_{0}}{S_{0}(\beta)+\bar{R}_{0}} \\
S_{0}(\beta) & =\sqrt{\bar{R}_{0}^{2}+2 \bar{\beta}}-\bar{R}_{0} .
\end{aligned}
$$

The corresponding zeroth order stress components are then defined by Eqns (16) and (18).

\section{The first order problem}

If $\varepsilon=0$, Eqn (44b) has a solution that can be written in terms of hyperbolic or exponential functions of $Y$ and in which $\beta$ appears only as a parameter. Many of the results of the Appendix in Ref. [10] therefore apply to the case where the first order heat flux is a function of time. In particular, we obtain:

$$
\begin{aligned}
\bar{Q}_{1}(\beta)= & S_{1}^{\prime}(\beta) \cosh \left(S_{0}\right)+\bar{Q}_{0}(\beta) S_{1}(\beta) \sinh \left(S_{0}\right) \\
\bar{T}_{1}(Y, \beta)= & \bar{Q}_{1}(\beta)\left\{\sinh (Y)-\left[S_{0}+\tanh \left(S_{0}\right)\left(1-S_{0} \tanh \left(S_{0}\right)\right)\right] \cosh (Y)\right\} \\
F(Y, \beta)= & -\frac{\bar{Q}_{1}(\beta)}{2}\left\{\left[S_{0}+\tanh \left(S_{0}\right)\left(1-S_{0} \tanh \left(S_{0}\right)\right)\right] Y \sinh (Y)-Y \cosh (Y)\right\} \\
\bar{P}_{1}(\beta)= & \frac{1}{\cosh \left(S_{0}\right)}\left[-\left(1+\frac{S_{0} \bar{\omega}}{2}\right) G\left(S_{0}\right)+\left(\frac{S_{0} \bar{\omega}}{2}\right) \frac{d^{2} G\left(S_{0}\right)}{d Y^{2}}\right] \\
& +\frac{\bar{Q}_{1}(\beta)}{2}\left[\frac{S_{0}\left(1-S_{0} \tanh \left(S_{0}\right)\right)}{\cosh ^{2}\left(S_{0}\right)}-\tanh \left(S_{0}\right)\right] \\
{\left[\bar{\omega}^{2} Y\right.} & -\bar{\omega}-Y] \frac{\mathrm{d}^{2} G(Y)}{\mathrm{d} Y^{2}}+2 \frac{\mathrm{d} G(Y)}{\mathrm{d} Y}-\left[\bar{\omega}^{2} Y+\bar{\omega}-Y\right] G(Y) \\
= & -\bar{Q}_{1}(\beta)\left(1-\bar{\omega}^{2}\right)\left[Y-\bar{\omega}^{2} Y+2 \bar{\omega}\right] Y \cosh (Y)
\end{aligned}
$$

from (A9, A11-A15) of Ref. [10] where $\bar{\omega}-\tanh \left(S_{0}\left(\beta_{0}(Y)\right)\right)=\tanh (Y)$. Substituting for $\bar{Q}_{0}(\beta), \bar{T}_{0}(0, \beta), \bar{Q}_{1}(\beta)$, $\bar{T}_{1}(0, \beta), \bar{P}_{1}(\beta)$ from Eqns $(47),(55),(57),(58),(60)$ into Eqn (53), we obtain:

$$
\begin{aligned}
\frac{\bar{R}^{\prime}\left(P_{0}\right) Y \bar{\omega}}{2 \cosh (Y)} G^{\prime \prime}(Y) & -\frac{\bar{R}^{\prime}\left(\bar{P}_{0}\right)}{\cosh (Y)}\left(1+\frac{Y \bar{\omega}}{2}\right) G(Y)= \\
& -\cosh (Y)\left[\left(Y+\bar{R}_{0}\right)^{2}+\left(Y+\bar{R}_{0}\right) \bar{\omega}(1-Y \bar{\omega})\right. \\
& \left.+\frac{\bar{R}^{\prime}\left(\bar{P}_{0}\right)}{2} \frac{Y(1-Y \bar{\omega})}{\cosh ^{2}(Y)}-\frac{\bar{R}^{\prime}\left(\bar{P}_{0}\right) \bar{\omega}}{2}\right] S_{1}^{\prime}\left(\beta_{0}(Y)\right) \\
& -\left[\left(Y+\bar{R}_{0}\right) \sinh (Y)+\bar{\omega}(1-Y \bar{\omega}) \sinh (Y)\right. \\
& \left.+\frac{\bar{R}^{\prime}\left(\bar{P}_{0}\right) \sinh (Y)}{2\left(Y+\bar{R}_{0}\right)} \frac{Y(1-Y \bar{\omega})}{\cosh ^{2}(Y)}-\frac{\bar{R}^{\prime}\left(\bar{P}_{0}\right) \bar{\omega}}{2\left(Y+\bar{R}_{0}\right)}\right] S_{1}\left(\beta_{0}(Y)\right) .
\end{aligned}
$$

Equations (61) and (62) constitute a pair of coupled ordinary differential equations to determine the unknown quantities $G(Y)$ and $S_{1}(\beta)$, given suitable initial conditions at $Y=0$. These two equations can be reduced to a single second order equation, using the procedure described in Section 4.1 of Eqn [8].

Results

This solution was used in plotting the curves labelled $\varepsilon=0$ in Figs 3-8 above. Good agreement was obtained between the full numerical solution at small $\varepsilon$ and the analytical solution, thus serving as a check on the numerical algorithm.

We also undertook an extensive comparison of the predictions of the above analytical solution for $\varepsilon=0$, and the approximate solution of $\mathrm{Li}$ and Barber [8]. The latter was found to be significantly in error for $S_{0}(\beta)>0.2$. 